\title{
Persistent hypoglossal artery with a contralateral hypoglossal canal venous lake: a case report
}

\author{
Nuno Vaz' ${ }^{1}, M D$, WL Poon ${ }^{2}$, FRCR, FHKCR, SS Cheng'2 FRCR, FHKCR \\ ${ }^{1}$ Center for Diagnostic Imaging, Barcelona Clinic Hospital, Barcelona, Spain \\ ${ }^{2}$ Department of Radiology and Imaging, Queen Elizabeth Hospital, Jordan, Hong Kong \\ * Corresponding author: poonwl@ha.org.hk
}

Hong Kong Med J 2019;25:399-400

https://doi.org/10.12809/hkmj177157

\section{Case report}

A 44-year-old woman presented to the emergency department in December 2014 with acute severe right-sided headache that began at the occipital region and spread to the right temporal and frontal regions. Pain was only partially relieved by analgesics. The patient had no history of altered mental state or focal neurological deficits. Her medical history was unremarkable except for a road traffic accident a few months previously ago with consequent right lower limb trauma. Neurological assessment revealed no gross abnormality. An urgent non-contrast brain computed tomography (CT) scan showed no intracranial haemorrhage or other abnormalities. Due to persistence of symptoms the patient underwent brain magnetic resonance (MR) imaging at a private centre. A small skull lesion was evident on the right basiocciput for which further imaging study was requested at our hospital. A contrast 3-T MR scan with angiography sequences revealed that the previously reported lesion

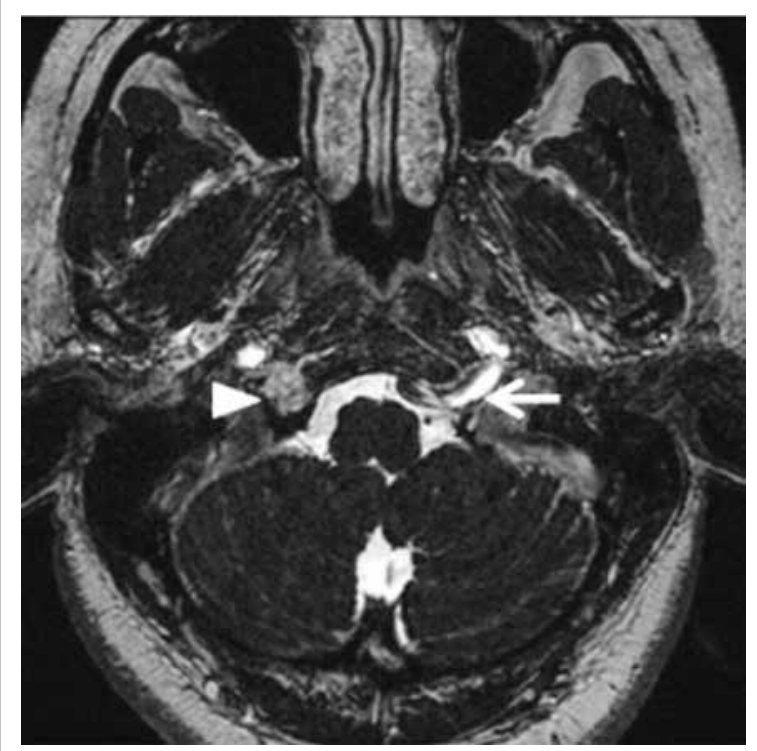

FIG I. Axial constructive interference in steady state magnetic resonance imaging delineated very clearly the left persistent hypoglossal (arrow) artery and the contralateral venous lake (arrowhead) corresponded to a $0.7-\mathrm{cm}$ T1-weighted isointense and T2-weighted hyperintense structure located at the right hypoglossal canal, which was expanded. It exhibited intense contrast enhancement and was in direct continuity with the inferior petrosal sinus and the internal venous plexus around the foramen magnum, all findings suggestive of a venous lake at the right hypoglossal canal (Fig 1).

Additionally, an anomalous vessel arising from the left internal carotid artery at C2 level was noted, entering the cranium through the left hypoglossal canal and joining the basilar artery. This anomalous vessel corresponded to a left persistent hypoglossal artery (PHA; Figs 1 and 2). The bilateral cervical



FIG 2. Straight anterior-posterior and left oblique projections of maximum intensity projection magnetic resonance angiography showing the left persistent hypoglossal artery (arrows) arising from the left cervical internal carotid artery 
vertebral arteries were diminutive in calibre and did not serve as major arterial supplies to the basilar artery. No intracranial aneurysms were detected and no infarction or other abnormality was noted. The patient's symptoms later substantially improved with symptomatic treatment.

\section{Discussion}

Bony venous lakes of the skull are common and asymptomatic, and they are typically parasagittal in location. In CT scans, they appear as lucent lesions with corticated/sclerotic margins. In MR imaging, they exhibit the same signal characteristics as veins. However, it is rare to find venous lakes located at the hypoglossal canal, and other entities must be excluded such as a neurinoma or even a dural arteriovenous fistula of the hypoglossal canal, another rare but potentially symptomatic condition that may follow head trauma. ${ }^{1}$ In this case, there was no apparent arteriovenous shunt detected in the MR angiography sequences.

A PHA results from failure of regression of a primitive hypoglossal artery, one of the several anastomoses that exist between the carotid and vertebrobasilar arteries during embryogenesis. Although rare, it is the second most common persistent carotid-vertebrobasilar anastomosis after the trigeminal artery, with a prevalence of up to $0.29 \%,{ }^{2}$ usually representing an incidental finding. However, diagnosis of PHA is important because it is often the only blood supply to the basilar trunk, as vertebral arteries are usually hypoplastic. Moreover, PHA is associated with intracranial arterial aneurysms, ischaemic cerebrovascular attacks, subarachnoid haemorrhage and arteriovenous malformations. ${ }^{3}$ Recognition of PHA is extremely important before any endovascular procedure, carotid endarterectomy or skull base surgery is performed. Exposure of the basilar trunk to an unusual haemodynamic stress could be the underlying mechanism that predisposes an individual to the development of aneurysms. ${ }^{4} \mathrm{On}$ the contrary, there is an increased risk of ischaemia caused by embolism from the internal carotid artery to the posterior circulation through the PHA. ${ }^{5}$

Both vascular anomalies in this patient were most likely incidental findings; however, owing to the reported association of PHA with intracranial aneurysm development and ischaemic events, any new episode or the development of neurological symptoms should have triggered immediate imaging study.

To the best of our knowledge, this is the first report of a PHA with a contralateral hypoglossal canal venous lake, both representing rare vascular variants.

\section{Author contributions}

All authors contributed to the concept of study, acquisition and analysis of data, drafting of the article, and critical revision for important intellectual content. All authors had full access to the data, contributed to the study, approved the final version for publication, and take responsibility for its accuracy and integrity.

\section{Conflicts of interest}

All authors have disclosed no conflicts of interest.

\section{Funding/support}

This case report received no specific grant from any funding agency in the public, commercial, or not-for-profit sectors.

\section{Ethics approval}

The patient was treated in accordance with the Declaration of Helsinki. The patient provided informed consent for all procedures.

\section{References}

1. Manabe S, Satoh K, Matsubara S, Satomi J, Hanaoka M, Nagahiro S. Characteristics, diagnosis and treatment of hypoglossal canal dural arteriovenous fistula: report of nine cases. Neuroradiology 2008;50:715-21.

2. Uchino A, Saito N, Okada Y, et al. Persistent hypoglossal artery and its variants diagnosed by CT and MR angiography. Neuroradiology 2013;55:17-23.

3. Srinivas MR, Vedaraju KS, Manjappa BH, Nagaraj BR. Persistent primitive hypoglossal artery (PPHA)-a rare anomaly with literature review. J Clin Diagn Res 2016;10:TD13-4.

4. Terayama R, Toyokuni Y, Nakagawa S, et al. Persistent hypoglossal artery with hypoplasia of the vertebral and posterior communicating arteries. Anat Sci Int 2011;86:5861

5. Conforto AB, de Souza M, Puglia P Jr, Yamamoto FI, da Costa Leite C, Scaff M. Bilateral occipital infarcts associated with carotid atherosclerosis and a persistent hypoglossal artery. Clin Neurol Neurosurg 2007;109:364-7. 